\title{
Nonparametric model research on price fluctuation of financial assets
}

\author{
Bailing Xu \\ Yunnan University Dianchi College, Kunming, Yunnan 650228, China \\ Email: xblxx2014@126.com
}

\begin{abstract}
Keywords: Financial assets; volatility; price fluctuation; nonparametric model
Abstract. From the perspective of non-parametric wave model, we focus on the nonparametric model and its application research. From nonparametric regression model, nonparametric autoregressive model, nonparametric VAR model family, nonparametric panel time series model. The above model estimation methods the development of nonparametric time series analysis is reviewed. This paper focuses on the nonparametric estimation techniques of nonlinear models, that is, the functional coefficient model, the nonparametric GARCH model family, and the predictions, tests and generalized impulse response functions. Finally, the characteristics of non-parametric time series analysis and the future development trend are pointed out. In this paper, the basic economic statistical theory and econometric methods will be adopted to study the development and application of nonparametric time series analysis. The author tries to use the relevant economic statistics theory to explain the real economic problems, and uses relevant econometric methods to describe. Verify economic theory on the interpretation of real problems, and then for the relevant departments of policy-making and risk early warning to provide a useful reference.
\end{abstract}

\section{Introduction}

Financial areas as the core of the modern market economy is the most intense competition Asian financial crisis swept Thailand, the Thai baht devalued [1-3]. Asia's rapid economic development boom was broken, some big countries began to enter the recession [4]. The political situation in some countries has also been affected, into a chaotic situation. China's capital account has not yet been opened by RMB, and the macroeconomic regulation and control that began in 1993 has already squeezed the dual protection of a large number of economic bubbles and survived the Asian financial crisis [5]. In 2007, due to the bankruptcy of subprime mortgage institutions and small and medium-sized banks, the investment fund was forced to close, and the severe shock caused the subprime crisis. The economic crisis caused the global major financial markets to become illiquid, which led to the global economic recession. The crisis in China has not survived, the impact of the direct performance of the subsequent 2008 decline in export trade, inflation, economic growth slowed significantly $[6,7]$.

In the context of interest rate market, interest rates are still rising stage, the domestic liquidity tightening situation makes the "money shortage" frequency is a dangerous signal [8]. As a major player in the credit market, capital markets, financial derivatives and commodities trading, "shadow banking" is a major player in the field of leveraged buyouts, which typically engages in lending, mortgaging, and leveraging large amounts of securities, bonds and complex financial instruments [9]. As a result of the blind spot in the government monitoring, bringing the financial market boom, while the rapid development of shadow banking and highly leveraged operations, with hidden deposit guarantee have nothing to fear, and may face the payment crisis and opaque non-standard operation are likely to become the future of the Chinese economy huge hidden dangers [10].

From the perspective of non-parametric wave model, we focus on the nonparametric model and its application research. From nonparametric regression model, nonparametric autoregressive model, nonparametric VAR model family, nonparametric panel time series model and the above model estimation methods the development of nonparametric time series analysis is reviewed. This paper focuses on the nonparametric estimation techniques of nonlinear models, that is, the functional coefficient model, the nonparametric GARCH model family, and the predictions, tests and 
generalized impulse response functions. Finally, the characteristics of non-parametric time series analysis and the future development trend are pointed out.

\section{Nonparametric regression model}

Consider a time series $\left\{Y_{t}\right\}_{t=1}^{n}$, the general non-parametric regression model is expressed as follows:

$$
\begin{gathered}
Y_{t}=f\left(X_{t}\right)+\sigma\left(X_{t}\right) \varepsilon_{t} \\
\hat{f}(x)=\frac{\sum_{t=1}^{n} Y_{t} K\left(h^{-1}\left(x-X_{t}\right)\right)}{\sum_{t=1}^{n} K\left(h^{-1}\left(x-X_{t}\right)\right)}
\end{gathered}
$$

Where $\mathrm{K}$ is a kernel function that satisfies:

$$
K(u) \geq 0, \int K(u) d u=1
$$

The form of kernel function includes homogeneous kernel, normal kernel, cubic kernel and Epanechnikov kernel. The choice of kernel function is not decisive to the final estimation result. Epanechnikov kernel is generally used in practical application. $\mathrm{H}$ is the window width, is to make the estimated mean square error of the minimum value, the choice of window width is used to balance the deviation and variance.

However, based on the study of the nonparametric econometric simultaneous equations model, the local linear tool variables estimation under certain conditions and the local linear tool variables with variable window width are given. The results show that the nonparametric VAR model can be used to solve the nonparametric VAR model. Estimation and other methods, we know that the VAR model itself is also a simultaneous model, which makes non-parametric VAR model estimates possible. The nonparametric SVAR model and its estimating method are given, and the conclusion is that the parameter SVAR model can explain the variables and do the corresponding impulse response and variance decomposition analysis, but the estimation precision and the prediction effect are lower than the non-parametric SVAR model.

The matrix form of a ternary nonparametric regression model:

$$
\begin{gathered}
Y_{t}=M\left(X_{t}\right)+\mu_{t} \\
Y_{t}=\left(y_{1 t}, y_{2 t}, y_{3 t}\right)^{\prime} \\
M=\left(m\left(X_{1 t}\right), m\left(X_{2 t}\right), m\left(X_{3 t}\right)\right)^{\prime} \\
\mu_{t}=\left(\mu_{1}, \mu_{2}, \mu_{3}\right)^{\prime}
\end{gathered}
$$

By introducing an instrumental variable $\mathrm{Z}$ such that it is related to the explanatory variable vector $\mathrm{X}$ but not to the random error term $\mathrm{t}$, the model can be estimated. The advantage of this method is that the model can estimate, estimate and predict the effect better than the parametric model by introducing the instrumental variables, and does not produce "dimension curse". The disadvantage is that the impulse response function of nonparametric SVAR model is not given at the moment, So that it can not do the appropriate impulse response analysis. However, since an approximation expression within each window width is available, the impulse response within each window width can be given from the definition of the impulse response function.

The local polynomial estimate is:

$$
\begin{gathered}
\widehat{f}(x)=\arg _{\gamma_{0}} \min _{\gamma_{0}, \cdots, \gamma_{t}} \sum_{i=1}^{n} \sum_{t=2}^{T}\left\{X_{(i) t}-\sum_{s=0}^{t} \frac{\gamma_{s}}{s !}\left(X_{(i) t-1}-x\right)^{s}\right\}^{2} K_{h}\left(X_{(i) t-1}-x\right) \\
\widetilde{f}(x)=\frac{1}{T-1} \sum_{t=2}^{T}\left\{\widetilde{f}_{t}(x)-\widetilde{f}_{t}(0)\right\}
\end{gathered}
$$


The BL model becomes a linear model with respect to $\varepsilon$, and when $\varepsilon$ is fixed, the BL model becomes a linear model with respect to tx and is therefore called a bilinear model. It can be seen that the BL model is actually a natural extension of the ARMA model in the nonlinear case. The BL model effectively utilizes the prediction residual error to correct the error feedback. In practical application, the BL model has a simple structure, few parameters and strong adaptability so it is a kind of nonlinear time series model with wide application.

\section{Construction of nonparametric ARFIMA model}

In the literature, the model is usually estimated and predicted directly after the model is given, but the model test is neglected. However, from the theoretical point of view, the model test is the same as the above model. The above non-parametric model is also necessary to test it, assuming the test form is as follows:

$$
H_{0}: a_{j}(u)=\beta_{j}\left(u, \gamma_{j}\right), j=1, \cdots, p
$$

Here $\beta$ is a specific form of function, for which the parameter vector. The test statistic takes the generalized likelihood ratio:

$$
T_{N, p^{\prime}}=\frac{N-p^{\prime}}{2} \log \left(\hat{\sigma}_{0}^{2} / \hat{\sigma}_{1}^{2}\right)
$$

To further test the fitting effect of the two models, monthly data from July to September 2012 are used as the forecast period (for convenience, the data are abbreviated as SZ and SC). Respectively, the two sets of data to take the number of different corresponding monthly rate of return sequence, as shown in Figure 1.

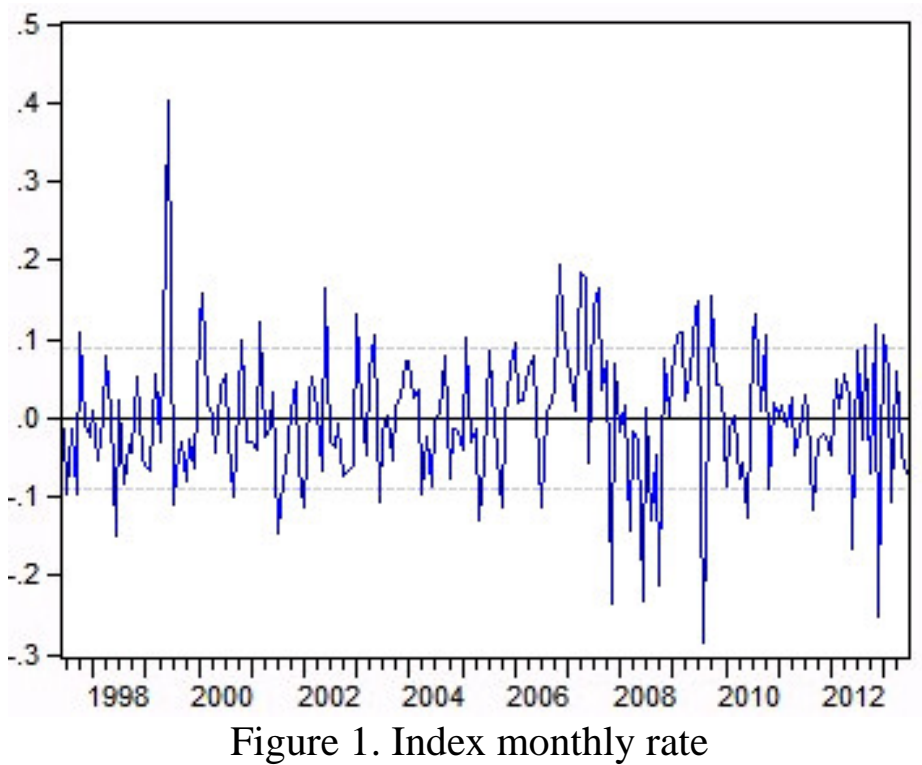

Shanghai composite index, Shenzhen stock index monthly yield data are in the vicinity of the shock, the Shanghai composite index monthly rate of return data volatility between -0.3-0.3, while the Shenzhen stock index monthly rate of return of the shock data slightly larger, in the 0.3-0.4 between two directions of the wave tends to be consistent.

\section{Data analysis and results}

A. Model identification and estimation

We get ARMA $(4,4)$ model and ARMA model $(4,2)$. The results of ARMA model and ARMA model are as follows: the ARMA model and the ARMA model are obtained by the autocorrelation coefficient and partial correlation coefficient chart. The monthly return of the Shanghai composite index model parameters obtained from the estimation equation:

$$
\begin{gathered}
w_{1 t}=-0.35 w_{1 t-1}-0.67 w_{1 t-2}-0.25 w_{1 t 3}+0.65 w_{1 t-4}+\mu_{1 t}+0.25 \mu_{1 t-1}+0.81 \mu_{1 t-2} \\
w_{1 t}=(1-L)^{0.1378} y_{1 t}
\end{gathered}
$$


The ARFIMA model is written as follows:

$$
\left(1+0.35 L+0.67 L^{2}+0.25 L^{3}+0.65 L^{4}\right)(1-L)^{0.1378} y_{1 t}=\left(1+0.25 L+0.81 L^{2}+0.25 L^{3}+0.97 L^{4}\right) \mu_{1 t}
$$

Shenzhen stock index monthly yield parameter model obtained from the following equation:

$$
w_{2 t}=-0.27 w_{2 t-1}-0.77 w_{2 t-2}-0.08 w_{2 t-3}+0.25 w_{2 t-4}+\mu_{2 t}+0.17 \mu_{2 t-1}+0.98 \mu_{2 t-2}
$$

Figure 2 for the non-parametric ARFIMA model estimates of the Shanghai and Shenzhen monthly yield residual scatter diagram. Nonparametric ARMA model this part of the tw estimate is obtained by programming with $\mathrm{R}$ software (see Appendix I for specific procedures). By choosing the minimum value of $\mathrm{s}$ for the monthly yield of the Shanghai composite index, the optimal window width $\mathrm{h}$ is chosen as 0.401 , the monthly return on the Shenzhen stock index s value of 2, the optimal window width h selected 0.901 , GCV value of 0.0094 at this time. It can be seen from the figure that the residual data of the Shanghai and Shenzhen stock markets fluctuate randomly in the range of $(0.3$, 0.4), but the residual of the Shanghai composite index is slightly larger than the residual of the Shenzhen component index.

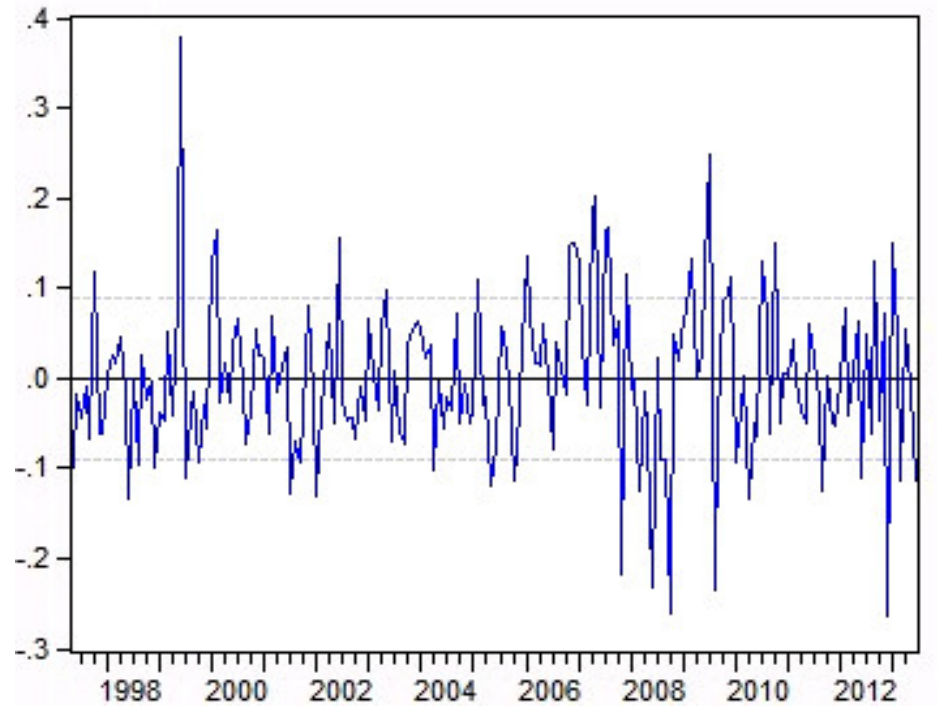

Figure 2. The non-parametric ARFIMA model estimates

\section{B. Stationary test}

Analysis of economic data in the establishment of the model before the general requirements of the data is stable, the use of the sequence ADF (unit root) test for stationary test, which can effectively avoid the model pseudo-regression phenomenon. The test results (Table 1) show that the difference data of REER, LnM2 and LnFDI are first-order stationary series at $1 \%$ level, and the weighted average interest rate $\mathrm{R}$ of inter-bank lending within 7 days rejects the null hypothesis at $5 \%$ Stationary sequence. The industrial added value data $\mathrm{Q}$ is a stationary series at the level of $10 \%$.

Table 1 . ADF test result of each variable

\begin{tabular}{lllll}
\hline Variable & ADF test - t value & significance level & $\begin{array}{l}p \\
\text { value }\end{array}$ & $\begin{array}{l}\text { Test } \\
\text { result }\end{array}$ \\
\hline REER & -0.69 & $-2.58^{* * *}$ & 0.84 & Unsteady \\
$\Delta$ REER & -6.88 & $-3.5^{*}$ & 0 & Steady \\
LnM2 & -0.32 & $-2.25^{* * *}$ & 0.92 & Unsteady \\
$\Delta$ LnM2 & -9.28 & $-3.5^{*}$ & 0 & Steady \\
LnFDI & -1.22 & $-2.58^{* * *}$ & 0.63 & Unsteady \\
$\Delta$ LnFDI & -11.17 & $-3.5^{*}$ & 0 & Steady \\
$Q$ & -2.33 & $-2.58^{* * *}$ & 0.09 & Steady \\
R & -4.3 & $-2.89^{* *}$ & 0.02 & Steady \\
\hline
\end{tabular}

Note: $\Delta$ is difference operator, $*$ is $1 \%$ significance level, ${ }^{* *}$ is $5 \%$ significance level, $* * *$ is significance level 


\section{Summary}

The author tries to use the relevant economic statistics theory to explain the real economic problems. Uses relevant econometric methods describe and verify economic theory on the interpretation of real problems, and then for the relevant departments of policy-making and risk early warning to provide a useful reference. From nonparametric regression model, nonparametric autoregressive model, nonparametric VAR model family, nonparametric panel time series model and the above model estimation methods the development of nonparametric time series analysis is reviewed. This paper focuses on the nonparametric estimation techniques of nonlinear models, that is, the functional coefficient model, the nonparametric GARCH model family, and the predictions, tests and generalized impulse response functions. Finally, the characteristics of non-parametric time series analysis and the future development trend are pointed out. In this paper, the basic economic statistical theory and econometric methods will be adopted to study the development and application of nonparametric time series analysis.

\section{References}

[1] Jeong D, Kim H, Park J Y. Does ambiguity matter? Estimating asset pricing models with a multiple-priors recursive utility[J]. Journal of Financial Economics, 2015, 115(2): 361-382.

[2] WangJue, WangRen, MiaoDuoqian, "Data enriching based on rough set theory", Journal of Environmental Sciences, vol. 29, no. 3, pp. 63-69, 2010.

[3] Zheng Zhonglong, Zhao Jianmin, Yang Jie. "NMF with Log Gabor Wavelets for visualization". Lecture Notesin Computer Scienee, vol. 3691, no. 2, pp. 26-32, 2005.

[4] Zhang Xiaodan, H u Feng , Zhao Li, "Improved Shuf F Led Frog Leaping Algorithm Based On Molecular Dynamics Simulations", Journal of Data Acquisition \& Processing, vol.27, no.3, pp.327-332, 2012.

[5] Rahimi-Vahed A, Mirzaei A H, "A Hybrid Multi-Objective Shuffled Frog-Leaping Algorithm For A Mixed Model Assembly Line Sequencing Problem", Computers and Industrial Engineering, , vol.53,no.4, pp.642-666, 2007.

[6] 3GPP TS 36.300, "Evolved Universal Terrestrial Radio Access (E-UTRA) and Evolved UTRA (E-UTRA)”, Dec. 2008. V8.0.0.

[7] Park H, Kim N, Lee J. Parametric models and non-parametric machine learning models for predicting option prices: Empirical comparison study over KOSPI 200 Index options[J]. Expert Systems with Applications, 2014, 41(11): 5227-5237.

[8] Rounaghi M M, Abbaszadeh M R, Arashi M. Stock price forecasting for companies listed on Tehran stock exchange using multivariate adaptive regression splines model and semi-parametric splines technique[J]. Physica A: Statistical Mechanics and its Applications, 2015, 438: 625-633.

[9] Zheng Z, Qiao Z, Takaishi T, et al. Realized volatility and absolute return volatility: A comparison indicating market risk[J]. PloS one, 2014, 9(7): e102940.

[10] Kalkuhl M, von Braun J, Torero M. Volatile and Extreme Food Prices, Food Security, and Policy: An Overview[M]//Food Price Volatility and Its Implications for Food Security and Policy. Springer International Publishing, 2016: 3-31. 